\title{
Frequency of Baker's Cysts in Cases of Meniscal Injury
}

\author{
Majed Alrowaili, M.D. \\ Department of Surgery, Faculty of Medicine, Northern Border University, Arar-91431, Saudi Arabia \\ majedalrowaili.nbu@gmail.com
}

\begin{abstract}
Magnetic resonance imaging (MRI) was used to investigate 394 subjects. Images were obtained in the three spatial orthogonal planes and evaluated by two expert radiologists. The state of meniscal and cruciate ligaments was recorded and statistical analysis was carried out by bivariate analysis. Out of twenty four of 394 subjects, 24 had Baker's cysts (6.2\%). Meniscal lesions were observed in 200 subjects (52\%), while 111 subjects (29\%) had a cruciate ligament lesion. Baker's cyst was found to have a statistically significant direct relationship with the presence and type of meniscal lesion $(P$-value $=0.01)$ but not with cruciate ligaments. The prevalence of Baker's cysts in the knees was in the acceptable range. The presence of Baker's cysts strongly correlated with the degree of meniscal injury.
\end{abstract}

Keywords: Knee injury, Baker's cysts, magnetic resonance imaging, meniscal injury.

\section{INTRODUCTION}

Popliteal cysts are the most frequently encountered cystic masses around the knee. They are the result of extravasation of joint fluid through a weakened posteromedial joint capsule between the medial head of gastrocnemius and the semimembranosus tendon into the gastrocnemius-semimembranosus bursa $[1,2,3,4]$ (figure1). Associations between popliteal cysts and internal derangement have been found even in the absence of an effusion [3].

The appearance of Popliteal or Baker's cyst is due to fluid distention of a bursa between the gastrocnemius and semimembranosus tendons [4]. Frequency of Baker's cysts identified by MRI ranges from $10 \%$ to $41 \%$ [5]. Baker's cysts may be seen with many joint abnormalities, such as internal derangement, osteoarthrosis, or inflammatory arthritis; the most common associations include joint effusion, meniscal tear, and degenerative joint disease [4].

The diagnosis of Baker's cyst is effectively made with MR imaging because fluid distention of the gastrocnemius-semimembranosus bursa is well depicted in $\mathrm{T}_{2}$-weighted axial MR images [4]. The purpose of this study was to evaluate the prevalence of baker's cysts (popliteal cysts) in patients using magnetic resonance (MR) imaging in Arar, Saudi Arabia and also to determine if the presence of Baker`s cysts was significantly associated with a Meniscal injury.
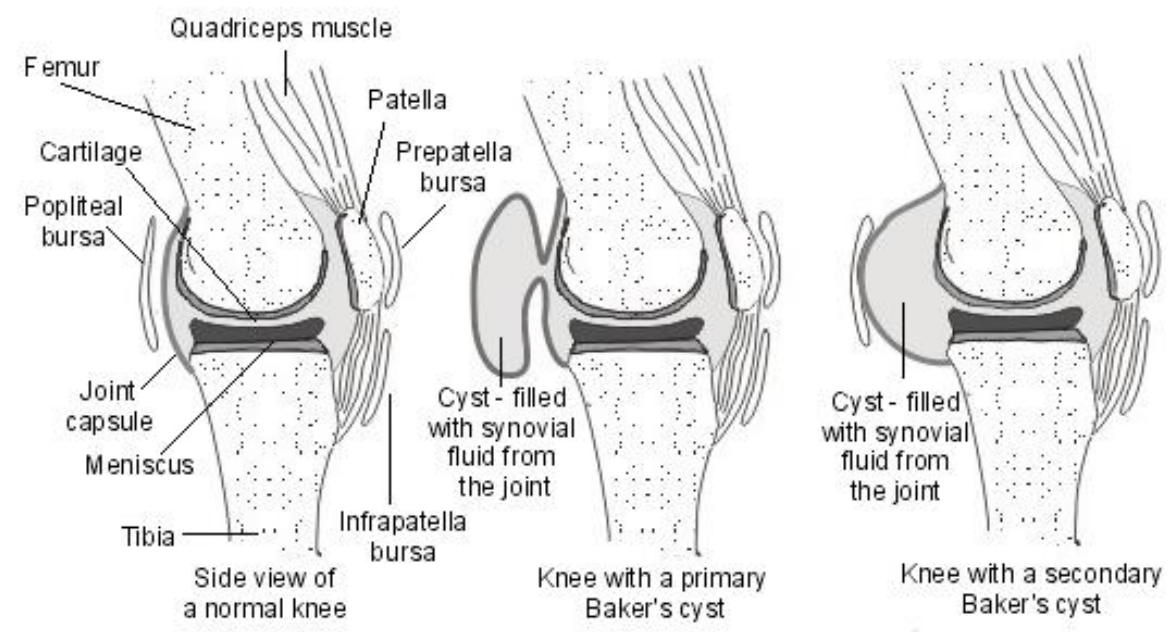

Figure1. Normal knee joint compared with abnormal knee joint showing the presence of Baker's cyst. 


\section{Materials \& Methods}

\subsection{Selection of Subjects}

A total of 394 subjects were studied by an MRI examination of the knee. MR images were prospectively evaluated by antwo expert two radiologists. Subjects were included when the orthopedic surgeon wanted to rule out the presence of meniscal, ligamentous or cartilage lesions. Out of these subjects, $83(21.6 \%)$ were female and $301(78.4 \%)$ male. The mean age was 35.4 years (standard deviation 12.58), with a range between 14 and 85 years. None of the subjects had rheumatoid arthritis, infection or acute trauma.

\subsection{Magnetic Resonance Imaging}

MR images were obtained with a cp or 8-chanel dedicated knee coil at $1.5 \mathrm{~T}$ (Magnetom Symphony; Siemens Medical Systems) with a standard protocol including PD-weighted frequency selective fat suppressed fast SE-sequences in coronal, sagittal and axial plane and T1-weighted coronal SE sequence with a slice thickness of 2.3 to $3.0 \mathrm{~mm}$ respectively. On MRI, the Baker's cyst was presented as a circumscribed mass with low signal in T1-weighed image, intermediate signal intensity in proton density (PD) image and high signal intensity when compared with skeletal muscle in PDweighed fat saturation image. A Baker's cyst was considered to be present when fluid collection was observed between the gastrocnemius and semimembranosus tendons in the transverse slices (figure2). No quantitative measurements of the synovial fluid or cyst content were made. Another recorded variable was the final clinical diagnosis.

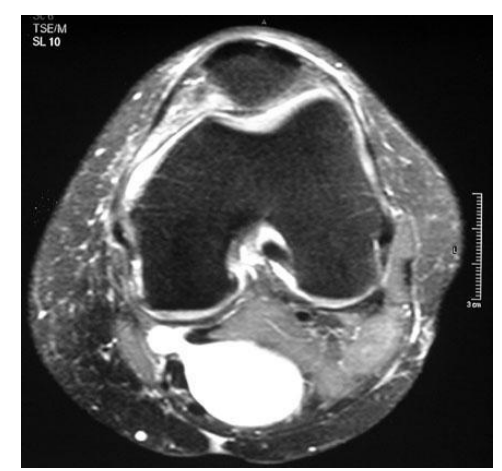

Figure2. Axial, T2-weighted magnetic resonance image with fat saturation reveals a Baker's cyst connected to the knee joint by way of a narrow neck between the tendons of the medial head of the gastrocnemius and semimembranosus muscles.

None of the subjects had received arthroscopy at the time opf study and no attempt was made to confirm the MR diagnosis. Pearson's chi-squared $(\chi 2)$ test was performed to compare proportions. The Gamma statistical analysis was also performed because both the files and columns of the tables had ordered values. Also, the means of the ages of two groups of patients (those with and those without Baker's cysts) were compared using a student's t-test. $\mathrm{P}$ value $=0.05$ was considered statistically significant.

\section{RESUlTS \& DiscuSSION}

Out of the 394 subjects, 83 (21.6\%) were female and 301 (78.4\%) male. The mean age was 35.4 years (standard deviation 12.58), with a range between 14 and 85 years. Baker's cysts were observed in 24 subjects (6.2\%), meniscal lesions were observed in 200 subjects (52\%), and 111 subjects (29\%) had cruciate ligament lesion. None of the subjects had rheumatoid arthritis, infection or acute trauma.

The relation between the Baker cyst presence and the Posterior Meniscus injury severity is expressed in Table 1. This relationship was statistically significant $(p=0.001$ The presence of Baker cysts was not statistically significantly related to severity of meniscal tear $(\mathrm{p}=0.84)$ Table 2 . The presence of cruciate ligaments lesions was statistically related with the Baker's cyst presence $(p=0.018$; Table 2$)$. Subjects with Baker cysts were older than those without (33 versus 32 years, respectively). This difference was found to not be statistically significant $(\mathrm{p}=0.93)$.

Table1

\begin{tabular}{|c|c|c|c|c|}
\hline & $\begin{array}{c}\text { Posterior Meniscus } \\
\text { injury Type 1 }\end{array}$ & $\begin{array}{c}\text { Posterior Meniscus } \\
\text { injury Type 2 }\end{array}$ & $\begin{array}{c}\text { Posterior Meniscus } \\
\text { injury Type 3 }\end{array}$ & Total \\
\hline Baker's cyst & 3 & 4 & 7 & 14 \\
\hline
\end{tabular}


Table2

\begin{tabular}{|c|c|c|c|c|c|}
\hline & $\begin{array}{c}\text { Anterior } \\
\text { Meniscus injury }\end{array}$ & $\begin{array}{c}\text { Posterior } \\
\text { Meniscus injury }\end{array}$ & $\begin{array}{c}\text { Cruciate Ligament } \\
\text { Injury }\end{array}$ & $\begin{array}{c}\text { Meniscus \& Cruciate } \\
\text { Ligament Injury }\end{array}$ & Total \\
\hline Baker's cyst & 3 & 14 & 3 & 4 & 24 \\
\hline
\end{tabular}

In MRI, Baker's cyst appears as a well-defined unilocular or multilocular cystic mass, located posteromedially between the tendon of the semimembranosus and the medial head of gastrocnemius [7-13]. Baker's cyst can be effectively diagnosed with MRI since the fluid-distented gastrocnemiussemimembranosus bursa is easily depicted on $\mathrm{T}_{2}$-weighted MR images, especially in the axial plane.

Fluid signal intensity is seen in all sequences in cases of popliteal cysts [11, 12]. Nevertheless, complications such as hemorrhage, rupture, the presence of intra-articular loose bodies and synovial proliferative reaction-diseases may produce a more heterogeneous appearance. Differential diagnosis from other cyst-like lesions and soft tissue tumors is thus imperative and requires a dedicated contrastenhanced MRI examination protocol [13-18].

Multiple studies have confirmed that intra-articular disorders have an important role in the pathogenesis of Popliteal cysts $[2,3]$. The prevalence of popliteal cysts increases with age, possibly due to degenerative thinning of the joint capsule [5]. The reported incidence of popliteal cysts on MR imaging is $4.7-19 \%$ in patients referred for internal derangement $[2,3]$. The clinical presentation of a Popliteal cyst is typically asymptomatic and rarely present for the evaluation of a cyst or mass. The presence of pain suggests internal derangement of the knee not by the cyst itself $[1,3,19]$. MRI examination can be used for determination of cyst contents and to reveal any associated internal disorder and also to differentiate other mass lesions.

Complications and differential diagnosis are most likely related to compression of adjacent structures or due to rupture and leakage [19]. The most important clinical differential diagnosis is thrombophlebitis, pseudophlebitis, compressing the popliteal vein hemorrhage or a calf hematoma with secondary compartment syndrome $[19,20]$ Other complications include direct compression of the popliteal artery with resultant ischemia, compression of adjacent nerves leading to entrapment symptoms, mechanical pressure symptoms, infection and hemorrhage [3, 19, 20] (Fig. 3) (Fig. 2).

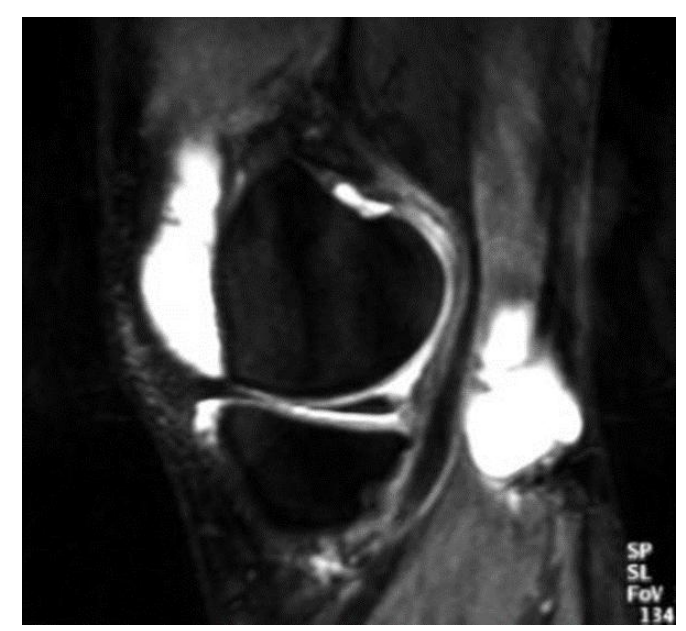

Figure3. Sagittal, $T_{2}$-weighted magnetic resonance image with fat saturation of the knee shows a large knee effusion and a Baker's cyst.

The management of uncomplicated cysts are usually treated conservatively [17]. Aspiration is sometimes undertaken, occasionally with instillation of corticosteroid. The steroid appears to be more effective when injected into the Baker's cyst directly compared to when it is injected directly into the joint [19]. Arthroscopic treatment of underlying knee arthropathy has had lasting resolution of an associated Baker's cyst. [22]

Indications for Baker's cyst excision include cases in which the popliteal cyst does not respond to conservative treatment or arthroscopic intervention or cases in which an underlying cause cannot be found [23]. Surgical excision is indicated primarily if the knee disorder is incurable and the cyst responsible for substantial local symptoms despite surgical closure is associated with a high recurrence rate $[19,20]$. 


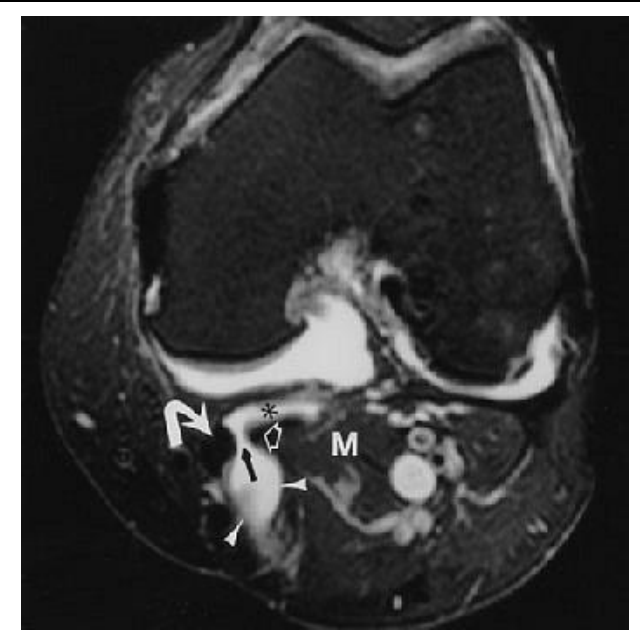

Figure4. Axial proton density-weighted MR image with fat saturation reveals Baker's cyst (arrowheads) with fluid (black arrow) between semimembranosus tendon (curved white arrow) and medial gastrocnemius (open arrow). Note the sub-gastrocnemius component (asterisk) of Baker's cyst. $M=$ medial gastrocnemius muscle.

Reference. Ward EE, Jacobson JA, Fessell DP, Hayes CW, and van Holsbeeck M, "Sonographic Detection of Baker's Cysts Comparison with MR Imaging" (AJR 2001; 176:373-380).

\section{CONClusion}

This study revealed that the prevalence of Baker's cysts the knees was in the acceptable range. The presence of Baker's cysts strongly correlated with the posterior meniscus injury. Furthermore, some correlation was also found between Baker's cysts and anterior meniscus and cruciate ligament injuries.

\section{REFERENCES}

[1] White EM. Magnetic Resonance imaging in synovial disorders and arthropathy of the knee. Magn Reson Imaging Clin North Am 1994;2:451-61 .

[2] Lindgren PG, Willen R. Gastrocnemio-semimembranous bursa and its relation to the knee joint. Anatomy and histology. Acta Radiol 1977;18:497-512.

[3] Fielding JR, Franklin PD, Kustan J. Popliteal cysts: a reassessment using magnetic resonance imaging. Skeletal Radiol 1991;20:433-5.

[4] Gristina AG, Wilson PD. Popliteal cysts in adults and children: a review of 90 cases. Arch Surg 1964;88:357-63.

[5] Miller TT, Staron RB, Koenigsberg T, Levin TL, Feldman F. MR imaging of Baker cysts: association with internal derangement, effusion, and degenerative arthropathy. Radiology 1996;201:247-250.

[6] Janzen DL, Peterfy CG, Forbes JR, Tirman PFJ, Genant HK. Cystic lesions around the knee joint: MR imaging findings. AJR 1994;163:155-161.

[7] MR imaging of cysts, ganglia, and bursae about the knee. Beaman FD, Peterson JJ Radiol Clin North Am. 2007 Nov; 45(6):969-82, vi.

[8] MR imaging of cysts, ganglia, and bursae about the knee. Beaman FD, Peterson JJ Magn Reson Imaging Clin N Am. 2007 Feb; 15(1):39-52.

[9] Cyst-like lesions of the knee joint and their relation to incident knee pain and development of radiographic osteoarthritis: the MOST study. Guermazi A, Hayashi D, Roemer FW, Niu J, Yang M, Lynch JA, Torner JC, Lewis CE, Sack B, Felson DT, Nevitt MC Osteoarthritis Cartilage. 2010 Nov; 18(11):1386-92.

[10] Janzen DL, Peterfy CG, Forbes JR, Tirman PF, Genant HK. Cystic lesions around the knee joint: MR imaging findings. AJR Am J Roentgenol. 1994;163:155-161.

[11] McCarthy CL, McNally EG. The MRI appearance of cystic lesions around the knee. Skeletal Radiol. 2004;33:187-209.

[12] Williams HJ, Davies AM, Allen G, Evans N, Mangham DC. Imaging features of intraosseous ganglia: a report of 45 cases. Eur Radiol. 2004;14:1761-1769. 
[13] Marra MD, Crema MD, Chung M, et al. MRI features of cystic lesions around the knee. Knee. 2008;15:423-438.

[14] Stacy GS, Kapur A. Mimics of bone and soft tissue neoplasms. Radiol Clin North Am. 2011;49:1261-1286.

[15] Magn Reson Imaging Clin N Am. 2009;17:489-507.

[16] Subhas N, Bui KL, Sundaram M, Ilaslan H, Recht MP. Incidental tumor and tumor-like lesions around the knee. Semin Musculoskelet Radiol. 2009;13:353-370.

[17] Chung CB, Boucher R, Resnick D. MR imaging of synovial disorders of the knee. Semin Musculoskelet Radiol. 2009;13:303-325.

[18] Papp DF, Khanna AJ, McCarthy EF, Carrino JA, Farber AJ, Frassica FJ. Magnetic resonance imaging of soft-tissue tumors: determinate and indeterminate lesions. J Bone Joint Surg Am. 2007;89(Suppl 3):103-115.

[19] Johnson LL, van Dyck GE, Johnson CA, Bays BM, Gully SM. The popliteal bursa (Baker's cyst): an arthroscopic perspective and the epidemiology. Arthroscopy 1997;13:66-72. L. Marti'Bonmat 210 ' et al. : Magnetic Resonance Materials in Physics, Biology and Medicine 10 (2000) 205-210.

[20] Miller TT, Staron RB, Koenigsberg T, Levin TL, Feldman F. MR imaging of Baker cysts: association with internal derangement, effusion, and degenerative arthropathy. Radiology 1996;201:247-50.

[21] Bandinelli F, Fedi R, Generini S, et al; Longitudinal ultrasound and clinical follow-up of Baker's cysts injection with steroids in knee osteoarthritis. Clin Rheumatol. 2012 Apr;31(4):727-31.

[22] Lie CW, Ng TP; Arthroscopic treatment of popliteal cyst. Hong Kong Med J. 2011 Jun;17(3):180-3.

[23] Snir N, Hamula M, Wolfson T, et al; Popliteal cyst excision using open posterior approach after arthroscopic partial medial meniscectomy. Arthrosc Tech. 2013 Aug 16;2(3):e295-8.

\section{AUTHOR's BIOGRAPHY}

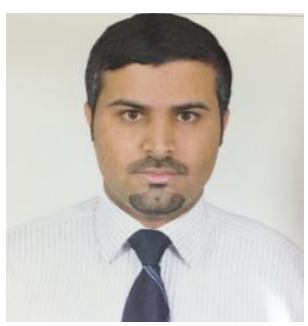

Dr. Majed Alrowaili, is currently serving as the Dean of Faculty of Applied Medical Sciences, Northern Border University and is also an Assistant Professor in the Department of Surgery, Faculty of Medicine, Northern Border University, Arar, Saudi Arabia. He passed the Saudi/Jordanian Board in Orthopedic Surgery in 2010. He has also completed a fellowship in Hands and Upper Extremities at Queens University, Canada and another fellowship in Orthopedic Sports Medicine, from Queens University, Canada in 2013. 